\title{
Multi-Approach Formulation in Dynamic Personality-Situation Interaction for Personnel Selection
}

\section{Mohd Hanafiah Ahmad, Gusman Nawanir, and Mohd Rashid Ab Hamid}

Faculty of Industrial Management, Universiti Malaysia Pahang, Lebuhraya Tun Razak, 26300 Gambang, Pahang, Malaysia

\section{Abstract}

The purpose of personnel selection is to measure knowledge, skills, and abilities that are necessary to perform a job effectively. The process involves various assessments, including personality assessment. This conceptual paper discussed the potential of using a learning factory to develop multiple simulations for assessment center activities in assessing personality in different situations. Although traditional personality assessment contributes to the effectiveness of selection decisions and prediction, it tended to ignore that trait-related behaviors may differ across situations. Study on dynamic personality is essential as empirical studies showed that within-person

Corresponding Author: Mohd Hanafiah Ahmad hanafiah@ump.edu.my

Received: 5 August 2019 Accepted: 14 August 2019 Published: 18 August 2019

Publishing services provided by Knowledge E

(c) Mohd Hanafiah Ahmad et al. This article is distributed under the terms of the Creative Commons Attribution License, which permits unrestricted use and redistribution provided that the original author and source are credited.

Selection and Peer-review under the responsibility of the FGIC2019 Conference Committee.

\section{G OPEN ACCESS}

fluctuations in personality states relate to a variety of work outcomes, including job performance. To further understand this fundamental issue, this paper discussed further how personality-situation interplay influences performance by using a learning factory assessment center method. This study also discussed how the adaptation of exploratory mixed methods approach could be used. The mixed exploratory methods are suitable as this topic is related to fundamental research and empirical study, besides the investigation on this area is still limited. This paper could benefit other researchers, industry players, and policymakers in understanding better how dynamic personality may influence performance, especially in the activities related to Industry 4.0 .

Keywords: personality dynamic, situation specific, assessment centre, learning factory.

\section{Introduction}

Personnel selection aims to assess whether a candidate has the knowledge, skills, and attitudes (KSA) required for a particular job. In this context, personality traits are among these KSA that being assessed. Typically, the selection procedure includes a wide variety of assessments, with personality assessment being a commonly used one (Ahmad, 2018; Lievens et al., 2018; Sosnowska, Brussel, Hofmans, Brussel, \& Lievens, 2018). Scholars suggest that personality assessment will add incremental value above and beyond general mental ability when predicting work performance (Judge \& 
Zapata, 2015). Furthermore, personality traits assessment also crucial for legal and social reasons as it shows little discrepancy as a function of ethnic or racial group (Roth, Bobko, \& Buster, 2013). Therefore, personality assessments are generally considered as a valid, reliable, and legally sustainable approach for assessing applicants' capabilities to perform effectively at work.

Although personality assessment contributed to the effectiveness of selection decisions, however, it ignores that trait-related behaviors may differ across contexts and also within one context across situations and time (Pulakos, Mueller-Hanson, \& Arad, 2019; Sosnowska et al., 2018). Instead of dismissing within-person fluctuations as measurement error, several scholars suggest that the lack of stability in behavioral manifestations is meaningful because it results from the interaction between personal characteristics and people's perceptions of the situation (Lievens et al., 2018). In the same vein, previous studies also show that local context across various situations may influence the view of fairness and acceptance of personnel selection process (Ahmad, 2018; Ahmad, Ab Hamid, \& Azizan, 2018).

This paper will discuss the potential of using a learning factory to develop multiple simulations for assessment center activities in assessing personality in different situations. Learning factory is a simulation factory to prepare students to be ready for industry application, including for industry 4.0. The suggestion to utilize learning factory method is in line with the National Policy on Industry Revolution 4.0 (Industry 4WRD) in which to enhance productivity, job creation and high-skilled talent pool in the manufacturing sector and its related services (Ministry of International Trade and Industry, 2018). However, in the context of Upskilling Existing and Producing Future Talents Industry 4.0, the policy focuses on knowledge and skills only. From a human resource management perspective, on top of these two elements, the focus should also be given on attitude and personality assessment. Therefore, the study on dynamic personality using learning factory may be useful to provide an empirical result to support that within-person fluctuations in personality states relate to a variety of work outcomes including job performance, especially for industry 4.0.

\section{Literature Review}

\subsection{Background of Personnel Selection}

Personnel selection typically focuses on evaluating candidates' performance related to knowledge, skills, and abilities (KSA) including their personality (Ahmad, 2018; Lievens 
et al., 2018; Sosnowska et al., 2018). To reach their goal, personnel selectors use prespecified procedures to assess whether the candidates have the required KSA to perform the job effectively. This goal creates an essential difference in personality research. In personality research, the focus of interest is on personality itself, typically on how and why personality is structured, developed, and manifested throughout life. Furthermore, personality research has also examined situations outside the job context in which, such as how employee making friends in new environments or how they resolve conflicts (Sosnowska et al., 2018). In this context, it shows that there is lacking integration between research on personnel selection and personality.

\subsection{Overview of Big Five Personality Assessment}

Big five personality or big five model factor is a model that has been developed to determine key human personality traits. According to Stidham, Summers, and Shuffler (2018), the Five-Factor Model (FFM) measures personality using five traits as represented by the OCEAN acronym: Openness, Consciousness, Extraversion, Agreeability, and Neuroticism. Openness relates to intellect, imagination, and independent-minded thinking. Being conscientiousness reflects orderly, responsible, and dependable people. Extraversion is typically characterized as being someone talkative, assertive, and energetic. Agreeableness is characterized as being good-natured, cooperative, and trustful. Finally, high levels of neuroticism show anxiety, being unhappy, and having negative emotions (Stidham et al., 2018). The personality Five-Factor Model (FFM) is the most prominent measure for the personality study as it has been validated for various populations, and both self-reports and peer evaluations.

\subsection{The Need to Improve Personality Assessment}

Several scholars argued that there are several reasons why the current personality assessment during personnel selection need to be improved.

The first reason is related to distortion in self-report personality inventories. Most of the previous studies found that self-reports of personality are vulnerable to intentional response distortion (Sackett, Lievens, Van Iddekinge, \& Kuncel, 2017). Such distortion has been shown to result in higher scores on job-relevant traits, lower standard deviations of scores, and higher inter-trait correlations. More important, fakers among candidates tend to rise to the tops of the score distributions, making likely better and honest candidates less likely to be selected (Sosnowska et al., 2018). Therefore, 
alternatives to traditional generalized self-report personality inventories that are less prone to faking good have been required.

The second reason is the need to incorporate work situations into personality assessment. Scholars have argued that incorporating work situations directly into personality assessment can improve the ability to hire the best applicants over-dependence on generalized personality self-report measures (Lievens, 2017b; Lievens et al., 2018). They further argued that contextualized personality inventories that add situational keywords and specify to candidates might improve prediction on their performance.

The last reason is related to candidates' perception toward selection procedures. Previous studies have found that how candidates view selection procedures affects their test motivation/test performance and may influence the validity of the selection procedure (Ahmad, 2018; Ahmad et al., 2018). Moreover, candidates use selection procedures as signals to make inferences about employer culture (Ahmad, 2018). In the same vein, meta-analysis research by Hausknecht, Day, and Thomas (2004) revealed that self-report personality inventories received an average favorability rating and work samples received among the highest ratings. Common reasons put forward by candidates for this relatively low rating include perceived low job-relatedness, rather impersonal nature compared to employment interviews, and lack of opportunity to demonstrate competence compared to work samples. Given lower favorability ratings of personality inventories, therefore, alternatives method is needed, which might be more favorably perceived by candidates.

\subsection{Dynamic Personality Assessment}

The perspective of dynamic personality focuses on the influence of situational and contextual factors on behavior. According to Sherman, Rauthmann, Brown, Serfass, and Jones (2015), the view of dynamic perspective holds the belief that traits are situation-bound, with psychologically active characteristics of situations triggering traitrelevant behavior. As opposed to the traditional trait method, the dynamic perspective recognizes that people respond in different ways to situational activates. It is not only in their behavior, feeling, and thinking, but also in other aspects like the variability of their behavior across situations in a different time. By taking into account the situationspecificity of personality displays, the dynamic perspective on personality has the potential to further improve the predictive validities of personality measures in personnel assessment (Sosnowska et al., 2018). 


\subsection{Assessing Dynamics Personality using Assessment Center Exercises}

Assessment center (AC) is a technique used in human resource management which involves multiple assessment processes, where a group of participants takes part in various exercises and is observed by a team of trained assessors who evaluate each participant against a number of predetermined, job-related behaviors (Ahmad, 2018; Martin Kleinmann \& Ingold, 2019; Thornton III, Rupp, Gibbons, \& Vanhove, 2019). These job-related behaviors are selected through job analysis and are deemed essential for successful performance in the target job. The effectiveness of a participant concerning these dimensions is evaluated in simulation exercises designed to replicate on-the-job situations. Although simulation exercises may differ considerably across $\mathrm{AC}$, the most commonly used are case analyses, in-baskets, oral presentations, role-plays, and group discussions. After finishing all the exercises, the judgments are pooled in a discussionbased meeting among assessors, by a statistical integration process, or a combination of both approaches (Ahmad, 2018; Martin Kleinmann \& Ingold, 2019; Thornton III et al., 2019).

Researchers have suggested that $A C$ can be used for various purposes in organizational settings. For example, Thornton, Rupp, and Hoffman (2014) provided examples of the use of $A C$ in recruitment, selection, placement, training and development, performance appraisal, organizational development, human resource planning, promotion and transfer, and redundancies. Generally, these AC tasks are also referred to as behavioral stimulations, which means that they resemble actual job-related tasks that should enable making predictions about applicants' proficiency in these job-related areas (Kleinmann et al., 2011; Sosnowska et al., 2018).

In the context of this paper, multiple assessments in $\mathrm{AC}$ exercises are proposed to be used in learning factory activities to represent different situations to measure candidates' personality-situation specific. Concerning dynamic personality-situation specific assessment, AC methodology can be used to measure dynamic personality in two different ways, as below:

\subsubsection{Assessing Within-Person Stability and Change Using Assessment Centre}

Traditionally, discrepancies in candidates' scores on the same dimensions across exercises were considered measurement error and were believed to weaken convergent 
and discriminant validity (Jackson, Ahmad, Grace, \& Yoon, 2011). However, more recent findings suggest that inconsistencies score recognized that people's behavioral inconsistency across assessment exercises might provide meaningful information about candidates (Sosnowska et al., 2018). Scholars suggest that this situation might indicate how applicants adjust their behavior to differing situational requirements (Gibbons \& Rupp, 2009; Sosnowska et al., 2018). They pointed out that consistency should not be expected from candidates' scores and suggested that researchers and practitioners should instead focus on incorporating different patterns of behavioral to get a better grip on the dynamics of how applicants' traits interact with the situations in the AC exercises and affect their performance. In this context, Lievens (2017a) suggested that methodologies that strongly resemble the multiple assessments in the $A C$ procedure have already been applied in personality research on within-person stability and change. He further suggests that it should be possible to develop an $A C$ to measure the full range of applicants' work-related variability.

\subsubsection{Assessing Person-Situation Interactions Using Assessment Cen- tre}

As $A C$ involves multiple assessments, it may also provide another way to study personality dynamics. The aim is then to focus on the overall pattern of behavioral responses in the $\mathrm{AC}$ exercises and on "if... then..."- contingencies, indicating under which situations an individual may engage or disengages in specific behaviors (Sosnowska et al., 2018). In the same veins, Gibbons and Rupp (2009) argued that connecting applicants' behavior with appropriate contexts allows examining their proficiency signs, which represent individual differences in successful performance-situation contingencies (e.g., effective communication of someone in one-on-one but not in group settings). Sosnowska et al. (2018) also suggested that to be able to study person-situation contingencies; it is necessary not only to measure candidates' behavior but also to measure the situational characteristics systematically. In this context, it is also important for the assessors to include ratings of situational characteristics alongside ratings of the candidate.

\subsection{Learning Factory Assessment Center as a Context or Situation}

\subsubsection{Overview of the Learning Factory Assessment Centre}

To reduce the shortage of talent to support Industry 4.0, higher learning institutions play an essential role in providing the required knowledge and skills (Razali Hassan \& 
Asnidatul Adilah Ismail, 2018). One of the approaches is by using the learning factory. Several universities in Malaysia has introduced a learning factory in their teaching and learning, including Universiti Malaysia Pahang (UMP). Faculty of Industrial Management (FIM), UMP, for instance, develop their learning factory to support student's competency development, especially in the area related to Industry 4.0. Based on real industry environment. Learning factory is a simulation of actual work with the aim is to transfer theoretical knowledge into a practical context and thus contribute to bridging the theorypractice gap. This learning style can be a useful approach when real-life training is costly and of some risk for companies (Tvenge \& Ogorodnyk, 2018). Workplace-related scenarios can be mapped through practical learning. The implementation of a learning factory is in line with the Malaysian policy on Industry 4.0 (Industry4WRD) targeting the high-skilled talent pool in the manufacturing sector (Ministry of International Trade and Industry, 2018).

Therefore, learning factory can be utilized to study dynamic personality by developing various activities and multiple assessments related to Industry 4.0. to assess candidates KSA and in different situation and context.

\subsubsection{Learning Factory Assessment Centre Activity Using Design Think- ing}

In designing learning factory activities related to Industry 4.0, the model of design thinking can be used. According to Lee (2019), design thinking is a way of identifying human needs and creating solutions using design tools and thinking. This method will utilize personal creativity, social interaction, and commercialization aspect. Design thinking has brought great attention to the business press and has been announced as a new problem-solving approach that is well adapted to the many business challenges in fostering innovation and growth (Liedtka, 2015).

Wrigley and Straker (2018) explained that there are several phases in developing design thinking activities. Liedtka, Salzman, and Azer (2017) specified the phases involved in the initial idea exploratory, which focuses on idea generation by collecting data to identify the issue and specific requirements. It is then followed by prototyping and testing. The final activity will involve a presentation and communication of the design. 


\section{Activity 1: Idea Generation.}

This phase involves producing various ideas that are possible to solve the problems that have been identified. Creating the broadest possible range of ideas is essential at this level. This activity will require the researchers to use their imagination and look beyond available solutions, which will lead to more innovative suggestions. This phase may include various innovation methods, including prototypes, brainstorming, mind mapping, and sketching.

\section{Activity 2: Prototyping}

Prototyping is essential as it provides new views and possible solution to the problems. It could range from post-it notes to a tangible product. However, the more realistic prototype to the requirement of the actual user, the better the feedback will be received for improvement. This activity will allow the team members to identify faults in their design activity and improve the design of their product.

\section{Activity 3: Presentation and Communication}

The primary purpose of presentation and communication is to get feedback related to the idea and prototypes created in the previous stages. It will allow design improvement based on users' experience, and more importantly, indicate whether the defined problems are addressed correctly.

The above design thinking activities will require candidates to face various situations and context (e.g., time, requirements, end-users). Therefore, from these activities, it may give valuable information on which traits are suitable for each activity, and how candidates change their personality traits to fit the situations. Consequently, a conceptual framework to assess the interaction between dynamic personality and learning factory AC using a learning factory is proposed in Figure 1. 


\subsubsection{Design Thinking Activities Assessment Using Behaviorally Anchored Rating Scale (BARS)}

To measure the candidates' performance during design thinking activities, the Behaviorally Anchored Rating Scale (BARS) is proposed to be used. BARS was initially developed to minimize the effect of construct-irrelevant variance in measuring job performance. Scholars explained that currently BARS has also been used for various purposes, including job interview performance, motivation, classroom teamwork and teaching evaluation practices (Lubbe \& Nitsche, 2019; Martin-Raugh, Tannenbaum, Tocci, \& Reese, 2016; Toumbeva, Ratwani, Diedrich, Flanagan, \& Uhl, 2018).

Critical incidents technique which involves subject matter experts (SME) can be used to develop BARS. This technique will be useful to identify required job behavior in a given in the work situations (Aguinis \& Solarino, 2019; Kell et al., 2017). Related to this, it is suggested that during the development of BARS, SME should be involved to identify criteria and develop performance standards that will be used to evaluate candidates during design thinking activities. As scholars reported that there is increasing attention to the importance of soft skills for 21st-century work environment (Hirsch, 2017); therefore, this paper suggests the following dimensions be assessed during design thinking activities. The dimensions are teamwork, communication, persuasion and negotiation, and leadership.

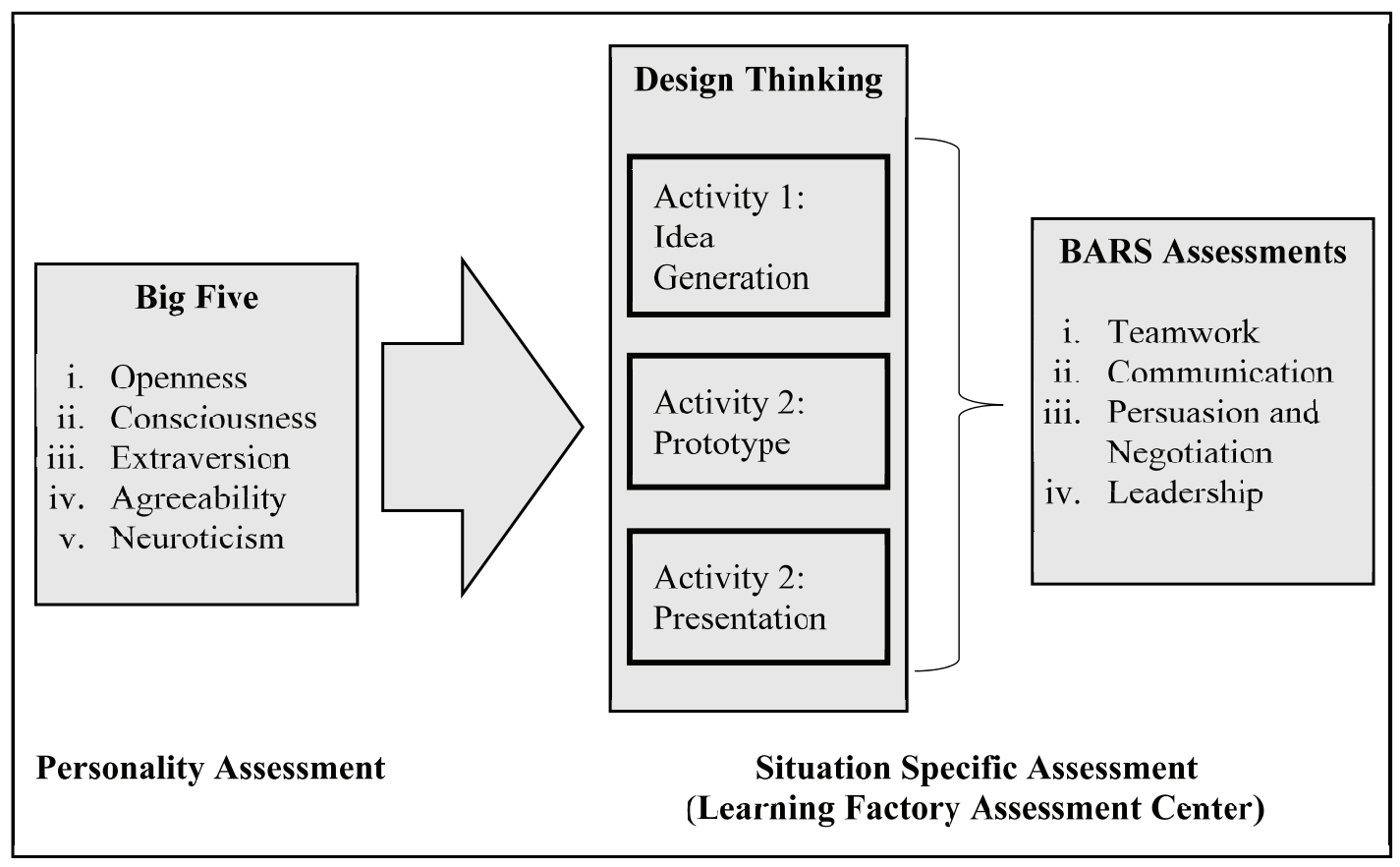

Figure 1: Conceptual Framework for Personality-Situation Specific Assessment (Source: Authors' work). 


\section{Dimension 1: Teamwork}

Kalisch and Lee (2009) defined the team as a group of people who support each other to attain a goal. As today's business environment is influenced by innovation and international competition. Therefore, this situation requires a good team culture in which they are not only responsible for performing their functions, but also to contribute to broader organizational success. The excellent teamwork environment is essential as the good teams are likely will achieve an organization's objectives than individuals (Jordan \& Ashkanasy, 2006). They are also more likely to generate more innovative ideas, which are essential for organizational success (Taylor \& Greve, 2006).

\section{Dimension 2: Communication}

Lee, Thomas, Zachariah, and Cooper (2019) explained that the form of communication could be in term of written communication, verbal communication, and non-verbal communication. In the $21^{\text {st }}$ century workplace, communication is consistently identified as a critically required skill (Carnevale, Smith, \& Strohl, 2013). More specifically, the empirical study found that listening and interpreting information is the most critical element in effective communication. Kell et al. (2017) explained that effective communication is not only by expressing ideas effectively and accurately but also as listening to the ideas and information provided by others. This skill is essential for teamwork because effective communication is needed to create strong group bonds.

\section{Dimension 3: Persuasion and Negotiation}

Zohar (2015) defined that negotiation is a way of settling conflicts between two or more parties. During this process, both sides need to deal with suggestions and counterproposals in order to achieve agreement. Negotiation and persuasion skills have often been identified as crucial to work success (Finegold \& Notabartolo, 2010). There are often arise issues in different perspectives, needs, and opinions when team member interacts with others. Hence, negotiation and convincing skills are needed to ease these differences (Kell et al., 2017). 


\section{Dimension 4: Leadership}

Leadership is widely used to facilitate change and is typically combined with authority power (Redmond \& Dolan, 2016). Leadership dimension is also regularly evaluated during selection interviews. Evaluation of leadership skill also includes motivating, coaching, meeting objectives, task delegation, and problem-solving (Huffcutt, Conway, Roth, \& Stone, 2001). Kell et al. (2017) commented that in recruiting future leaders, it is critical to evaluate leadership skill.

\subsection{Situation Strength and Proposition Development}

As design thinking activities in learning factory, AC will involve various situation, and it is expected that situation strength will influence how dynamic personality of the candidates. Judge and Zapata (2015) suggested that situation strength represents the degree to which situational restrictions are present in the work setting, including rules, structures, and expected behavior. In contrast, weak situations contain environments in which social roles are unstructured, organizational structures are decentralized, and the job provides considerable discretion with limited external control over one's behaviors. Meanwhile, central to weak situations is that the situation where context is vaguely structured. Concerning the conceptual framework as Figure 1, this paper suggests the following proposition:

Proposition 1: The relationship between conscientiousness, emotional stability, extraversion, agreeableness, and openness with job performance will be stronger in activities in which situation strength is required (i.e., the output of the work is delivered, and the process of how the work is done is clear).

Proposition 2: The relationship between conscientiousness and job performance relationship is stronger for activities that require independence and strong attention-todetail requirements.

Proposition 3: The relationship between emotional stability and job performance is stronger for activities require strong social skills.

Proposition 4: The relationship between extraversion and job performance is stronger for activities that require strong social skills and competitive requirement.

Proposition 5: The relationship between agreeableness and job performance is stronger for activities that require strong social skills and weaker in activities require a competitive requirement. 
Proposition 6: The relationship between openness and job performance is stronger for activities require independence and innovative requirements.

\section{Methodology}

Given the fact that study related to dynamic personality-situation specific is still limited, it is suggested that sequential exploratory mixed methods approach should be used. The study should start with a qualitative method and then followed with a quantitative method. According to Creswell (2008), this research approach is suitable to discover a phenomenon as it begins with the qualitative study and then followed with a quantitative approach. Scholars also explain that exploration is needed because guiding theories or framework are not fully established, or due to instruments, variables, or measures are unknown and not available.

\subsection{Qualitative Research Design}

The focus of the qualitative stage is to identify core competencies (knowledge, skills, attitude) required for job success for Industry 4.0. The initial stage of the study should start with a case study method. The case study will involve a series of in-depth data collection from various sources using different techniques including interviews and focus group discussions with subject matter experts, and document/website reviews (Creswell \& Poth, 2017).

In developing interview protocol to explore the required core competencies for Industry 4.0, research by Erol, Jäger, Hold, Ott, and Sihn (2016) and Nørgaard and Guerra (2018) can be used as guidance. These scholars suggested four groups of competencies, namely personal competencies, social competencies, action competencies, and domain competencies.

Based on feedback from the initial interviews, the research team can develop learning factory $A C$ activities, assessment methods and performance standards related to Industry 4.0 based on the design thinking model (Elsbach \& Stigliani, 2018; Garrette, Phelps, \& Sibony, 2018). As mentioned previously, design thinking model is suitable for this study as it involves idea generation, creativity, problem-solving, prototyping, testing, and presentation. Therefore, it will allow various activities to be designed which involve different contexts, situations and involve various interactions (between individuals, within groups, between groups). 
These multiple assessment activities should be later presented to the subject matter experts from industries. The purpose of this process is to verify the assessment activities, assessment methods, and performance measurement standards are suitable to measure related KSA in Industry 4.0 environment.

\subsubsection{Respondent for Qualitative Research}

In-line with the case study approach, a purposeful sampling technique should be applied to select the research participants. Guided by its protocols, a series of face-to-face and focus group interview need to be carried out to the selected companies who involve or in the stage of adapting Industry 4.0. Interview with officer-in-charge for Industry 4.0 in the Ministry of International Trade and Industry (MITI) should also be conducted. This is to understand the fundamental and implementation of Industry 4WRD policy further.

\subsubsection{Qualitative Data Analysis}

This qualitative data collection will generate two types of qualitative data (i.e., interviewer field notes, transcripts of interviews, focus group discussions, and document/website reviews). Subsequently, the qualitative data will be analyzed by using content analysis procedure following the analysis spiral suggested by Creswell and Poth (2017), which includes data managing; reading and memoing; describing, classifying, and interpreting; and finally representing and visualizing the data. The qualitative data analysis software Atlas.ti 8 will assist the process of data analysis.

\subsection{Quantitative Research Design}

After the qualitative data analysis, the quantitative phase should be conducted. By using the assessment center approach, this quantitative study aims to get data related to personality-situation interaction (Lievens et al., 2018; Sosnowska et al., 2018). To perform this activity, it will involve two groups; the first group is candidates that will involve in learning factory assessment center activities using the design thinking method. The second group will be the assessors that will involve in observing and recording personality-situation interaction for each candidate in each activity. 


\subsubsection{Respondent for Quantitative Research}

For the first group, a total of 30 candidates need to be recruited to perform multiple activities. The candidates will be divided into five groups consist of four candidates per group. This number is suitable enough to ensure interaction among candidates, and the assessors will be able to manage and observe the candidates during the activities. The candidates will perform three activities related to the design thinking model.

The second group is the assessors. For each activity, two assessors will be assigned for each group and will involve in observing and recording personality-situation interaction for each candidate.

\subsubsection{Measurement Development}

In the second stage of the study, three sets of measurements will be used. The first set of measurement is using existing Big Five personality test. The second and third set is a measurement that will be developed based on the results of the qualitative stage with support from the relevant previous studies. The second measurement focuses on dynamic personality - situation interaction, which will be answered by the candidates after each activity. The third set of measurement is an evaluation of dynamic personality - situation interaction by the assessors for each candidate in each activity.

In order to enhance the content validity and reliability, the instrument should be reviewed by a group of specialists in the area of industrial psychology, operations management, and Industry 4.0 and pre-test should be conducted. This process will include consultations and interviews with the respondents to examine the following aspects of the questions, (1) whether there are any questions that need to be included or excluded, (2) whether the content of the questionnaire is sufficient, (3) whether the right questions being asked, and (4) whether the questions are easy to understand. Also, a pilot test will be conducted in order to ensure the construct validity before the dissemination of the instrument in the real study. The feedback from the respondents from both pre-test and pilot test will be used to develop the better instrument by clarifying the wordings, and some measurement items might be added, discarded, or modified. 


\subsubsection{Quantitative Data Collection}

The quantitative study will start with assessing respondents' personality using the Big Five Model (Tasselli, Kilduff, \& Landis, 2018). This self-assessment is to record their personality without any situation interaction.

The next process will involve learning factory assessment centers activities. During these activities, respondents will be required to perform the activity and respond to the questions related to personality in a particular situation and activity. The purpose of this process is to gather data related to personality-situation interaction. In the same time, assessors will record data related to personality-situation interaction for each candidate.

\subsubsection{Data Analysis}

Three phases of quantitative data analysis need to be conducted in the second stage of the study. In the first phase, analysis of personality traits from self-respond Big Five personality test. In the second phase, the relationships among the variables can be examined by using the Structural Equation Modeling (SEM) approach with SmartPLS 3.2.8. Subsequently, based on the qualitative and quantitative findings; a finalize model of dynamic personality - situation interaction can be developed. The diagram in Figure 2 summarized suggested research flow for this study.

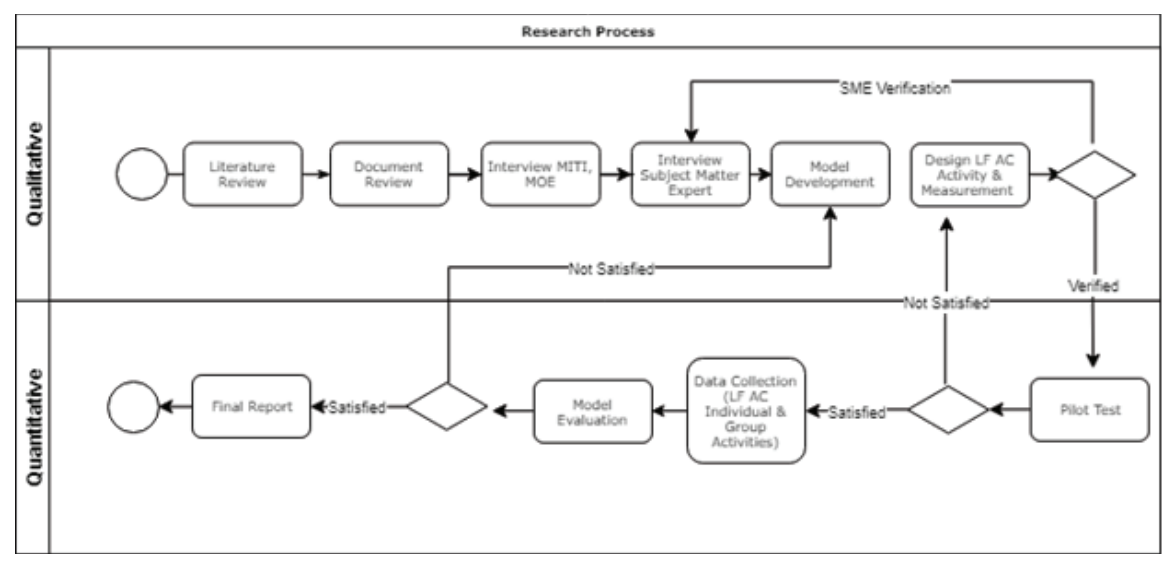

Figure 2: Suggestion of Research Flow for Personality-Situation Specific Assessment (Source: Authors' work).

\section{Discussion}

This conceptual paper aims to provide fundamental knowledge and research framework on personality dynamics in different situations, specifically in the context of Industry 
4.0 by using the assessment center approach and learning factory. As research on personality dynamics in Industry 4.0 is still new, therefore it will provide huge room for exploration and contribute to new knowledge and theory expansion.

As this study focuses on exploring the fundamental issue of why people change their behavior in response to situations, it is hoped that findings from this research will contribute to the expansion of the related theory (e.g., trait activation theory) by exploring interaction during learning factory assessment center activities will influence personality and behavior.

\section{Conclusion and Implications}

As the focus of Industry 4WRD policy is more on knowledge and skills, this study will enhance the policy by incorporating attitude assessment from a personality perspective. Besides that, the findings serve as a platform to design talent assessment program in a more comprehensive way by not only focusing on knowledge and skills related to Industry 4.0 but also personality and behaviors of the candidates. This will help employers to improve their human resource activities (e.g., selection, development, promotion).

\section{Acknowledgement}

We would like to thank Faculty of Industrial Management and FIM's Governance and Integrity Centre, Universiti Malaysia Pahang for the financial support by sponsoring this paper to be presented in the FGIC 2nd Conference on Governance and Integrity 2019.

\section{References}

[1] Aguinis, H., \& Solarino, A. M. (2019). Transparency and replicability in qualitative research: The case of interviews with elite informants. Strategic Management Journal.

[2] Ahmad, M. H. (2018). The effect of cultural factors on the design and acceptance of assessment centre practices in malaysian public sector. Universiti Malaysia Pahang.

[3] Ahmad, M. H., Ab Hamid, M. R., \& Azizan, N. A. (2018). Impact of culture on the acceptance and outcome of assessment centre method. Journal of Quality Measurement and Analysis JQMA, 14(1), 23-43. 
[4] Carnevale, A. P., Smith, N., \& Strohl, J. (2013). Recovery: Job growth and education requirements through 2020. Georgetown University Center on Education and the Workforce.

[5] Creswell, J. W. (2008). Education research: Planning, conducting and evaluating quantitative and qualitative research (Vol. 3rd). New Jersey: Pearson Education.

[6] Creswell, J. W., \& Poth, C. N. (2017). Qualitative inquiry and research design: Choosing among five approaches. Sage publications.

[7] Elsbach, K. D., \& Stigliani, I. (2018). Design thinking and organizational culture: A review and framework for future research. Journal of Management, 44(6), 22742306.

[8] Erol, S., Jäger, A., Hold, P., Ott, K., \& Sihn, W. (2016). Tangible Industry 4.0: a scenariobased approach to learning for the future of production. Procedia CiRp, 54, 13-18.

[9] Finegold, D., \& Notabartolo, A. S. (2010). 21st century competencies and their impact: An interdisciplinary literature review. Transforming the US Workforce Development System, 19-56.

[10] Garrette, B., Phelps, C., \& Sibony, O. (2018). Structure and Solve the Problem Using Design Thinking. In Cracked it! (pp. 169-195). Springer.

[11] Gibbons, A. M., \& Rupp, D. E. (2009). Dimension consistency as an individual difference: A new (old) perspective on the assessment center construct validity debate. Journal of Management, 35(5), 1154-1180.

[12] Greene, J. C., Carecelli, V. J., Graham, W. F., Tashakkori, A., Teddlie, C., Greene, J. C., ... Graham, W. F. (2008). Toward a conceptual framework for mixed-method evaluation designs. In V. L. Plano Clark \& J. W. Creswell (Eds.), The mixed methods reader (pp. 121-150). Thousand Oaks, Ca: Sage.

[13] Hausknecht, J. P., Day, D. V, \& Thomas, S. C. (2004). Applicant reactions to selection procedures: An updated model and meta analysis. Personnel Psychology, 57(3), 639-683.

[14] Hirsch, B. J. (2017). Wanted: Soft skills for today's jobs. Phi Delta Kappan, 98(5), $12-17$.

[15] Huffcutt, A. I., Conway, J. M., Roth, P. L., \& Stone, N. J. (2001). Identification and meta-analytic assessment of psychological constructs measured in employment interviews. Journal of Applied Psychology, 86(5), 897.

[16] Jackson, D. J. R., Ahmad, M. H., Grace, G. M., \& Yoon, J. (2011). An alternative take on research and practice: Task-based assessment centers. In N. Povah \& G. C. Thornton (Eds.), Assessment Centres and Global Talent Management. Surrey: Gower Publishing. 
[17] Jordan, P. J., \& Ashkanasy, N. M. (2006). Emotional intelligence, emotional selfawareness, and team effectiveness. Linking Emotional Intelligence and Performance at Work: Current Research Evidence with Individuals and Groups, 145-163.

[18] Judge, T. A., \& Zapata, C. P. (2015). The person-situation debate revisited: Effect of situation strength and trait activation on the validity of the Big Five personality traits in predicting job performance. Academy of Management Journal, 58(4), 1149-1179.

[19] Kalisch, B. J., \& Lee, H. (2009). Nursing teamwork, staff characteristics, work schedules, and staffing. Health Care Management Review, 34(4), 323-333.

[20] Kell, H. J., Martin-Raugh, M. P., Carney, L. M., Inglese, P. A., Chen, L., \& Feng, G. (2017). Exploring methods for developing behaviorally anchored rating scales for evaluating structured interview performance. ETS Research Report Series, 2017(1), 1-26.

[21] Kleinmann, M., \& Ingold, P. V. (2019). Toward a Better Understanding of Assessment Centers: A Conceptual Review. Annual Review of Organizational Psychology and Organizational Behavior, 6, 349-372.

[22] Kleinmann, M., Ingold, P. V, Lievens, F., Jansen, A., Melchers, K. G., \& Konig, C. J. (2011). A different look at why selection procedures work: The role of candidates' ability to identify criteria. Organizational Psychology Review, 1(2), 128.

[23] Lee, H. (2019). Revitalising Traditional Street Markets in Rural Korea: Design Thinking and Sense-Making Methodology. International Journal of Art \& Design Education, 38(1), 256-269.

[24] Lee, S. W. H., Thomas, D., Zachariah, S., \& Cooper, J. C. (2019). Communication Skills and Patient History Interview. In Clinical Pharmacy Education, Practice and Research (pp. 79-89). Elsevier.

[25] Liedtka, J. (2015). Perspective: Linking design thinking with innovation outcomes through cognitive bias reduction. Journal of Product Innovation Management, 32(6), 925-938.

[26] Liedtka, J., Salzman, R., \& Azer, D. (2017). Design thinking for the greater good: Innovation in the social sector. Columbia University Press.

[27] Lievens, F. (2017a). Assessing personality-situation interplay in personnel selection: Toward more integration into personality research. European Journal of Personality, 31(5), 424-440.

[28] Lievens, F. (2017b). Integrating situational judgment tests and assessment centre exercises into personality research: Challenges and further opportunities. European Journal of Personality, 31(5), 487-502.

[29] Lievens, F., Lang, J. W. B., De Fruyt, F., Corstjens, J., Van de Vijver, M., \& Bledow, R. (2018). The predictive power of people's intraindividual variability across situations: 
Implementing whole trait theory in assessment. Journal of Applied Psychology, 103(7), 753.

[30] Lubbe, D., \& Nitsche, A. (2019). Reducing assimilation and contrast effects on selection interview ratings using behaviorally anchored rating scales. International Journal of Selection and Assessment.

[31] Martin-Raugh, M., Tannenbaum, R. J., Tocci, C. M., \& Reese, C. (2016). Behaviorally anchored rating scales: An application for evaluating teaching practice. Teaching and Teacher Education, 59, 414-419.

[32] Ministry of International Trade and Industry. (2018). Industry 4WRD. National Policy on Industry 4.0. Kuala Lumpur.

[33] Nørgaard, B., \& Guerra, A. (2018). Engineering 2030: Conceptualization of Industry 4.0 and its implications for Engineering Education. In 7 th International Research Symposium on PBL (p. 34).

[34] Pulakos, E. D., Mueller-Hanson, R., \& Arad, S. (2019). The Evolution of Performance Management: Searching for Value. Annual Review of Organizational Psychology and Organizational Behavior, (0).

[35] Razali Hassan, \& Asnidatul Adilah Ismail. (2018). Challenge and issues in human capital development towards industry revolution 4.0. In Proceedings of International Conference on The Future of Education IConFEd) 2018. Pulau Pinang: Institute of Teacher Education Tuanku Bainun Campus, Penang, Malaysia,.

[36] Roth, P. L., Bobko, P., \& Buster, M. A. (2013). Situational judgment tests: The influence and importance of applicant status and targeted constructs on estimates of B lack-W hite subgroup differences. Journal of Occupational and Organizational Psychology, 86(3), 394-409.

[37] Sackett, P. R., Lievens, F., Van Iddekinge, C. H., \& Kuncel, N. R. (2017). Individual differences and their measurement: A review of 100 years of research. Journal of Applied Psychology, 102(3), 254.

[38] Sherman, R. A., Rauthmann, J. F., Brown, N. A., Serfass, D. G., \& Jones, A. B. (2015). The independent effects of personality and situations on real-time expressions of behavior and emotion. Journal of Personality and Social Psychology, 109(5), 872.

[39] Sosnowska, J., Brussel, V. U., Hofmans, J., Brussel, V. U., \& Lievens, F. (2018). Assessing personality dynamics in personnel selection. In J. F. Rauthmann (Ed.), The handbook of personality dynamics and processes. Elsevier.

[40] Stidham, H., Summers, J., \& Shuffler, M. (2018). Using the Five Factor Model to Study Personality Convergence on Student Engineering Design Teams. In DS92: 
Proceedings of the DESIGN 2018 15th International Design Conference (pp. 21452154).

[41] Tasselli, S., Kilduff, M., \& Landis, B. (2018). Personality change: Implications for organizational behavior. Academy of Management Annals, 12(2), 467-493.

[42] Taylor, A., \& Greve, H. R. (2006). Superman or the fantastic four? Knowledge combination and experience in innovative teams. Academy of Management Journal, 49(4), 723-740.

[43] Thornton, G. C., Rupp, D. E., \& Hoffman, B. J. (2014). Assessment Center Perspectives for Talent Management Strategies. New York: Routledge.

[44] Thornton III, G. C., Rupp, D. E., Gibbons, A. M., \& Vanhove, A. J. (2019). Samegender and same-race bias in assessment center ratings: $A$ rating error approach to understanding subgroup differences. International Journal of Selection and Assessment.

[45] Toumbeva, T. H., Ratwani, K. L., Diedrich, F. J., Flanagan, S. M., \& Uhl, E. R. (2018). Development of a Behaviorally Anchored Rating Scale for Leadership. APTIMA INC WOBURN MA WOBURN United States.

[46] Tvenge, N., \& Ogorodnyk, O. (2018). Development of evaluation tools for learning factories in manufacturing education. Procedia Manufacturing, 23, 33-38.

[47] Wrigley, C., Mosely, G., \& Tomitsch, M. (2018). Design Thinking Education: A Comparison of Massive Open Online Courses. She Ji: The Journal of Design, Economics, and Innovation, 4(3), 275-292.

[48] Zohar, I. (2015). "The Art of Negotiation" Leadership Skills Required for Negotiation in Time of Crisis. Procedia-Social and Behavioral Sciences, 209, 540-548. 